\title{
Orbital metastasis as the initial presentation of lung adenocarcinoma: a case report
}

\author{
This article was published in the following Dove Press journal: \\ OncoTargets and Therapy \\ 9 May 2016 \\ Number of times this article has been viewed
}

\author{
Liangchao Sun ${ }^{1,2, *}$ \\ Yali $\mathrm{Qi}^{1,3, *}$ \\ Xindong Sun ${ }^{2}$ \\ Jinming $\mathrm{Yu}^{2}$ \\ Xue Meng² \\ 'Medical College of Shandong \\ University, ${ }^{2}$ Department of Radiation \\ Oncology, Shandong Cancer Hospital \\ Affiliated to Shandong University, \\ Shandong Academy of Medical \\ Science, Jinan, ${ }^{3}$ Department of \\ Oncology, The Second Hospital of \\ Lanzhou University, Lanzhou, People's \\ Republic of China \\ *These authors contributed equally \\ to this work
}

\begin{abstract}
Orbital metastasis as the initial presentation of lung adenocarcinoma is very rare, and so the lack of knowledge about this phenomenon can easily result in misdiagnosis, either as a orbital primary tumor or benign lesion. Here, we report a rare case in which the orbital symptom appeared first without any pulmonary manifestations. Our patient developed decreasing vision in his right eye over a 3-month duration. He then presented with proptosis and multiple aches from head to back. After systemic evaluation, our patient was diagnosed with Stage IV non-small-cell lung cancer and was managed with palliative chemoradiotherapy. The final result of treatment suggests that the therapeutic efficacy of chemotherapy on orbital metastasis is uncertain, and only some orbital metastatic masses may have a favorable response to radiation. Furthermore, we review the recent data and provide an in-depth discussion on the clinical features and course of ocular pulmonary metastases, and explain a new type of non-small-cell lung cancer metastatic pattern for ophthalmologists and oncologists to help them distinguish the orbital metastasis as the first manifestation.
\end{abstract}

Keywords: orbital metastasis, skeletal metastasis, rare metastasis, initial presentation, lung adenocarcinoma

\section{Introduction}

It is estimated that more than 1.6 million new cases of cancer were diagnosed in 2015 , of which lung or bronchus cancer accounted for $14 \%$ of cancer in men, and $13 \%$ in women. About one-quarter (27\%) of all cancer deaths are due to lung cancer. More than one half $(57 \%)$ of the cases are diagnosed at a later stage, with a 5 -year survival rate of approximately $4 \% .{ }^{1}$ Current analysis confined to late-stage lung adenocarcinoma provides a better understanding of the metastatic sites including bone $(39 \%)$, brain $(25 \%)$, liver $(16 \%)$, and adrenal glands $(13 \%){ }^{2}$ Symptoms that generally cause the need for medical attention are the primary tumor, metastatic spread, or paraneoplastic syndrome. For the symptoms related to the distant metastatic spread as the first manifestation, diagnosis would be a challenge. When a metastatic disease is suspected, complete physical examination and history, standard laboratory testing, adequate imaging, and endoscopic biopsies are required to assess the clinical staging. Patients with distant metastatic disease are usually managed with systemic therapy (chemotherapy, targeted therapy, or a combination), clinical trials, or palliative treatment. However, metastases to some other sites have been reported rarely in the literature, especially the initial spread to the orbit, and the diagnosis, treatment, and prognosis of this is also not reported often. In our case, orbital metastasis was the first manifestation that attracted medical attention. The incidence, diagnosis, and treatment are discussed in this paper.
Correspondence: Xue Meng

Department of Radiation Oncology, Shandong Cancer Hospital and Institute, No 440 Jiyan Road, Jinan, Shandong 2501 I 7 , People's Republic of China

Tel +8653 I 67626142

Fax +86 53। 6762 6I4I

Email mengxue5409@126.com (c) (1) (5) 2016 Sun et al. This work is published and licensed by Dove Medical Press Limited. The full terms of this license are available at https://www.dovepress.com/terms.php

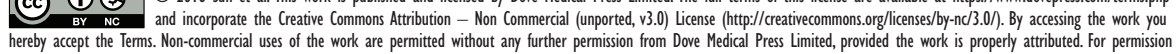
hereby accept the Terms. Non-commercial uses of the work are permitted without any further permission from Dove Medions.
for commercial use of this work, please see paragraphs 4.2 and 5 of our Terms (https://www.dovepress.com/terms.php). 


\section{Case report}

A 33-year-old man was referred to the eye clinic with a chief complaint of progressively decreasing vision in his right eye. The patient reported that he first noted the change in vision 3 months previously, without any other remarkable features. He also reported that when he moved his right eye, he felt a sense of discomfort and aching pain. One month later, a small palpable, round, movable, nontender bump was found in his left chest wall, which was getting bigger in size from $2 \times 2 \mathrm{~cm}^{2}$ to $3 \times 3 \mathrm{~cm}^{2}$ in recent months. Excluding the change in vision and the bump in the chest wall, further detailed ophthalmic examination and general physical assessment showed no significant abnormalities. He had no history of trauma or any other underlying systemic and ophthalmologic disease. He had a smoking history of 10 pack-years and a history of alcohol use. A cranial and nasopharynx computed tomography (CT) showed a right solid tissue orbital mass in the retrobulbar space, pressing on the optic nerve; there was no involvement of the bony orbital wall, and the brain was normal. The initial clinical impression was that of orbital displacement, and it was difficult to differentiate the benign tumors (capillary hemangioma, optic nerve glioma, cavernous hemangioma, and meningioma), inflammatory pseudotumor, or malignant tumor, all of which show a similar unclear shadow on plain scan. Before further investigative examinations were ordered, the ophthalmologist recommended the patient to a surgical clinic for evaluation of left chest wall neoplasm to determine if it was a primary malignancy or metastasis. A subcutaneous resection was performed, and the histopathology examination of the excision showed poorly differentiated metastatic adenocarcinoma. Furthermore, immunohistochemical studies showed negativity of TTF-, NapsinA, S-100, HMB45, and Melan-A and positivity of CK, CK7, and Vim. Four weeks after his first doctor visit, the patient came to our radiation oncology clinic, complaining of decreasing visual acuity, proptosis of his right eye and aching pain without lid ptosis or other relevant focal neurological deficits in contrast to the left eye, and onset of multiple aches from head to back. The laboratory tests showed cytokeratin fragment antiogen 21-1: $181.5 \mathrm{ng} / \mathrm{mL}$ (normal, 0.0-3.3) and neuron-specific enolase: $72.97 \mathrm{ng} / \mathrm{mL}$ (normal, $0-17$ ). On the basis of the findings, his left chest wall neoplasm was regarded as a metastasis from an unknown primary source, and there was a large suspicion that the retrobulbar mass was also a metastasis. An investigative systemic oncologic workup was initiated. In the chest CT, an enhancing mass (Figure 1) was found in the lower lobe of the right lung, with right hilum and mediastinal lymph node metastases, and enhancements in multifocal muscular neoplasms (Figure 2), suggestive of metastatic involvement. A CT-guided percutaneous fine-needle aspiration biopsy (FNAB) of the lung mass was performed, and it provided a histopathological diagnosis of adenocarcinoma, which is consistent with the known subcutaneous tumor (Figure 3). By this time, visual acuity and pain in the right eye progressed in severity. A spontaneous subcapsular hemorrhage was observed in the right eye. A diagnostic magnetic resonance imaging (MRI) of the nasopharynx was repeated, and it demonstrated multifocal skeletal muscular metastases and a right retrobulbar extraconal mass $\left(20 \times 15 \times 20 \mathrm{~mm}^{3}\right)$ that was increasing in size, pushing the eyeball forward (Figure 4A), and two enhancing nodules in brain (Figure 5A). The experienced medical radiologist reported that all the lesions showed similar patterns on different series, highly supportive for the metastatic involvements. A bone scan showed no abnormal values. After a multidisciplinary discussion with the physicians (including oncologists, radiologists, and surgeons), we did not perform an invasive biopsy because of the high risk of complications, which would further affect his potential vision because of the close anatomical relationships, as well as the patient's poor condition and refusal. According to the clinical, radiological, and histopathological evidence, our patient was considered to have stage IV (T2N2M1) non-small-cell lung cancer (NSCLC)
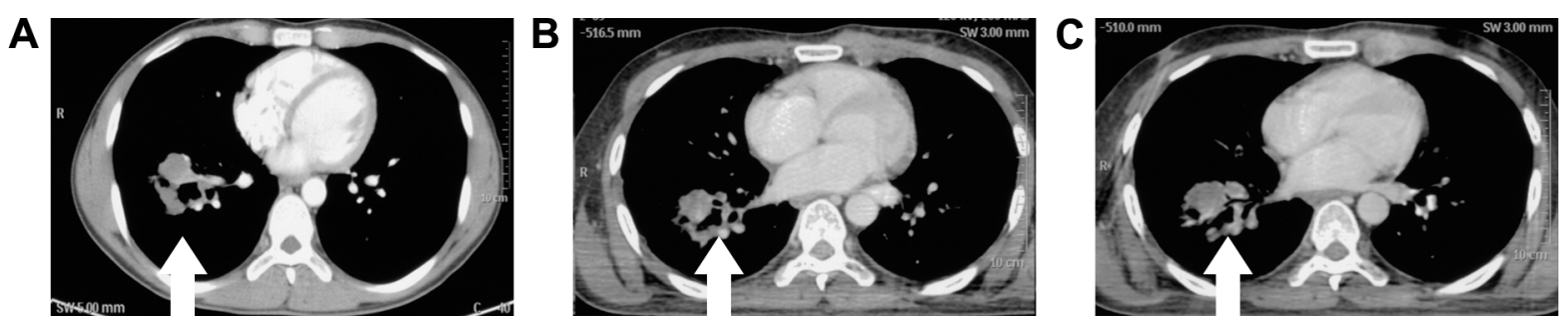

Figure I Chest CT imaging.

Notes: (A) The image shows an enhancing mass $\left(42 \times 40 \mathrm{~mm}^{2}\right)$ in the lower lobe of the right lung before the treatment; white arrow indicates the primary lesions. (B) The figure shows that the pulmonary lesions (white arrow) had little response to the first-line chemotherapy. (C) It reveals progression of the disease (white arrow) after the second-line chemotherapy.

Abbreviation: CT, computed tomography. 

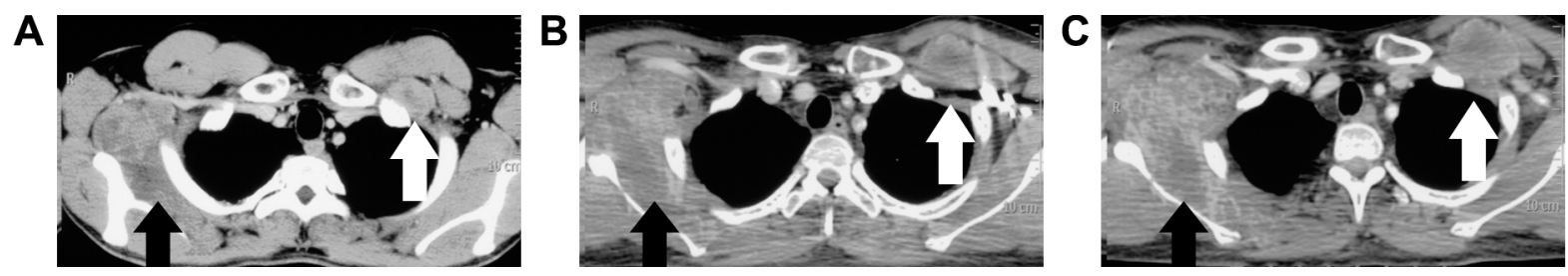

Figure 2 CT of the chest.

Notes: (A) CT shows enhancing masses in right subscapularis (black arrow) and pectoralis (white arrow) muscles before the treatment. (B) CT reveals an enlargement of the masses (white and black arrows) after first-line chemotherapy and (C) an increase in size of the mass (white arrow) after second-line chemotherapy and an stable subscapularis mass (black arrow) for the radiotherapy.

Abbreviation: $\mathrm{CT}$, computed tomography.

with brain, orbital, subcutaneous, and multifocal skeletal metastases. Once the diagnosis was certain, the patient was referred to the special ward for the palliative chemoradiotherapy. Tyrosine kinase inhibitors (TKIs) were denied as a first-line treatment because the histopathology report from our biopsy was negative for epidermal growth factor receptor (EGFR). He received first-line chemotherapy with two cycles of pemetrexed and cisplatin; in addition, palliative radiotherapy, 50 Gy in 25 fractions to relieve the pain and the decreasing visual acuity, was performed on the retrobulbar mass. In a repeated MRI scan, the orbital metastasis showed a significant response to the treatment (Figure 4B) and two more lesions were seen in the brain (Figure 5B). Because of the progression of the neoplasm to the brain, radiation to the whole brain was initiated, 50 Gy in 25 fractions. During the second cycle of chemotherapy, the skeletal muscular metastases enlarged rapidly, with pain escalating out of medical control (Figure 2B). Being judged as a process of progression according to the Response Evaluation Criteria In Solid Tumors (RECIST), our patient underwent second-line chemotherapy with docetaxel and cisplatin. Additionally, radiation to the skeletal muscle metastases was initiated. When the treatment was completed, he reported relief from

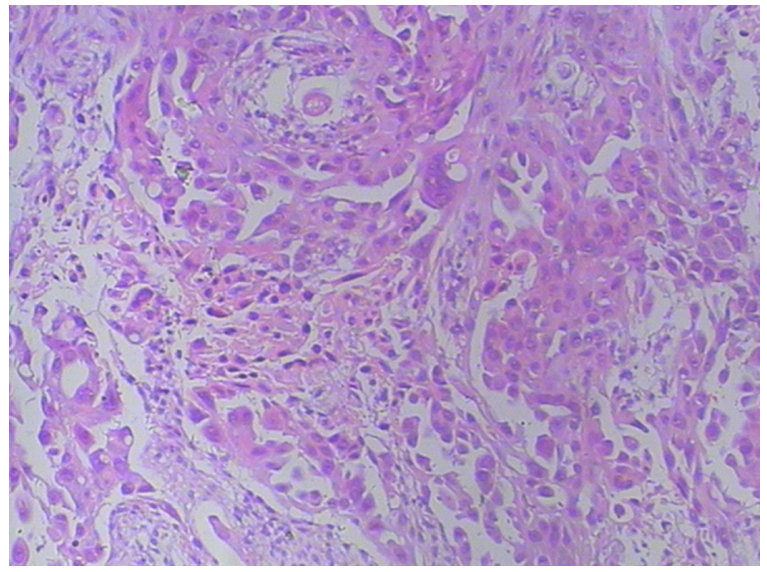

Figure 3 Histopathologic study of the CT-guided biopsy from the lung mass, showing poorly differentiated adenocarcinoma (H\&E, $\times 400)$.

Abbreviations: $\mathrm{CT}$, computed tomography; H\&E, hematoxylin and eosin. the pain in the metastatic sites and improvement in visual acuity (Figure 4C). No relevant side effects of radiotherapy were found by CT scan and physical examinations. The patient was subsequently lost to follow-up.

This study was approved by the Institutional Review Board of Radiation Oncology, Shandong Cancer Hospital and Institute. The patient provided written informed consent.

\section{Discussion}

Although rare, orbital symptoms can be the initial clinical presentation preceding the diagnosis of the primary silent lung neoplasm. At the beginning, the ophthalmologist failed to detect this metastasis possibly because of the rarity of such a presentation. We believe that our case can provide a special insight into the metastatic patterns of lung cancer and should be of great assistance to the clinician in formulating a differential diagnosis when a patient has suspected orbital metastasis at presentation. Orbital metastases of cancer are rare and comprise about $3 \%-7 \%$ of orbital lesions and about $10 \%$ of orbital tumors. They most commonly originate from primary lesions of the breast $(39 \%-48 \%)$, prostate and skin (melanoma) (12\%), lung (8\%), and kidney (7\%-11\%) in whites. With the increasing number of cases of lung cancer, the probability that a physician will see a patient with an orbital metastasis is also increasing. ${ }^{3-5}$ No matter from where the primary tumor originates, orbital metastases generally cause a further reduced vision due to alteration of ocular motility and the invasion of optic nerve. ${ }^{6}$ From a review of the literature, we find that features vary in different areas in the world. The percentage of orbital symptoms as the first manifestation with unknown primary cancer in most series is about $15 \%$ in contrast with the $52.17 \%$ reported in the People's Republic of China. ${ }^{7-9}$ Previous studies show that the majority of tumors are usually unilateral, with the average age of onset from 40 to 60 years..$^{7-10}$ The possibility of malignant tumors increases with age, since older patients are more prone to metastasis. Occurrence of tumors in women is higher than that in men in whites, but the reverse is found in Japan and South China. An appropriate explanation for 

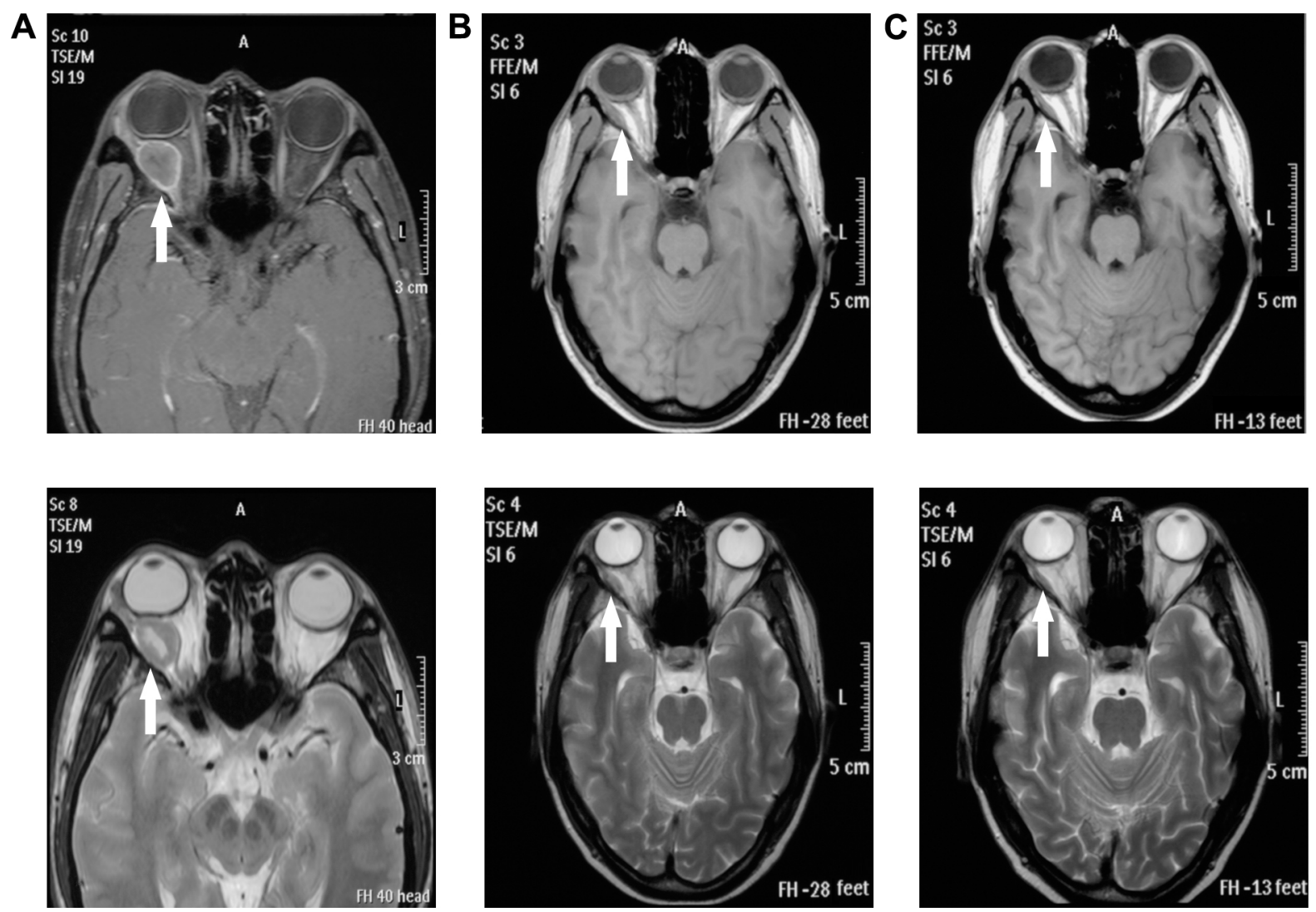

Figure 4 Orbital mass secondary to metastatic lung cancer on axial, enhanced, TI-weighted, and T2-weighted MR images.

Notes: (A) Before treatment, a right retrobulbar extraconal mass $\left(20 \times 15 \times 20 \mathrm{~mm}^{3}\right)$ (white arrow) is shown pressing on the optic nerve, and a severe globe proptosis can be noticed. (B) A significant reduction of the intraorbital mass (white arrow) can be found after administration of a radiation dose of $30 \mathrm{~Gy}$. (C) The mass (white arrow) decreases in size significantly after administration of the whole radiation dose of $50 \mathrm{~Gy}$. The proptosis and visual acuity is evident on the MRI scan.

Abbreviation: MRI, magnetic resonance imaging.

this distinction may result from the quite different origin of the primary tumor; in whites, breast cancer causes the first orbital metastasis, while in Japan and South China, lung cancer and nasopharyngeal cancer cause the most orbital metastases. ${ }^{8,11}$ The most frequent pathological type is adenocarcinoma (65\%), and more than half of them are of a poorly differentiated type, similar to what was observed in our patient. ${ }^{12}$ Another interesting finding is the timing of ocular involvement. The symptoms had been present for about 2 months prior to the initial diagnosis, partially because any expansion of a mass in the restricted orbital volume would result in symptoms manifesting much sooner than a similar
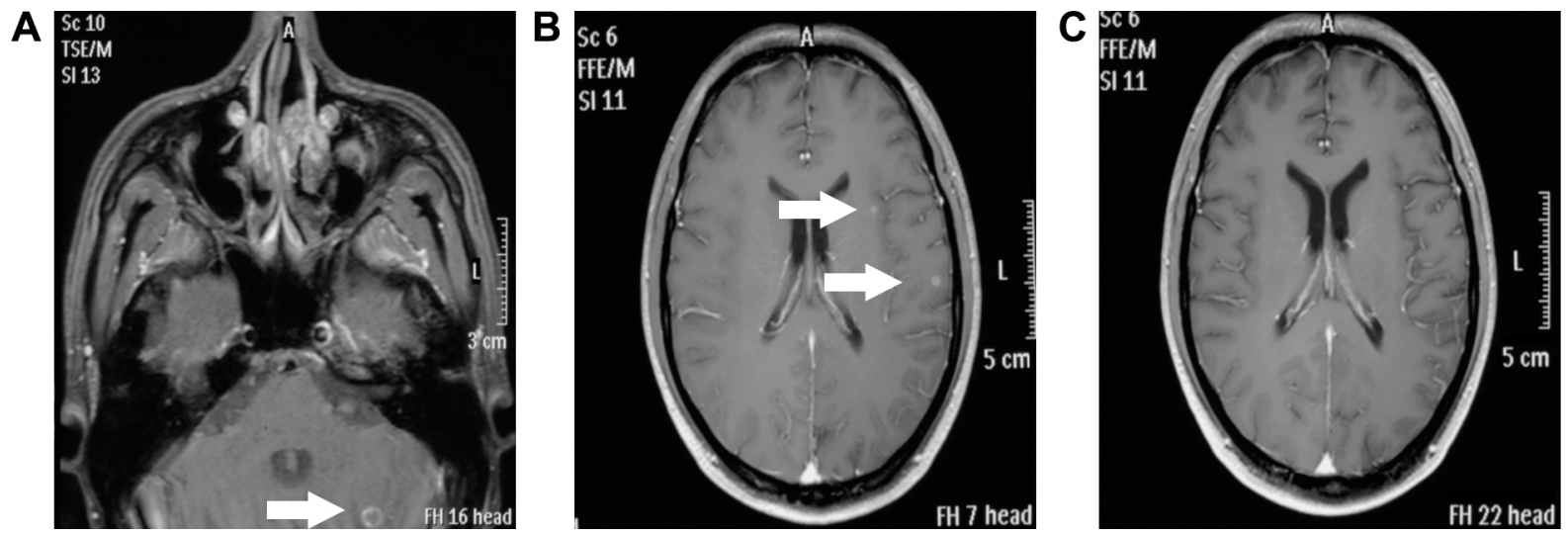

Figure 5 Brain metastases on the MR images.

Notes: (A) Metastasis in the left cerebellar hemisphere (white arrow) and left temporal lobe (not shown). (B) Two new lesions (white arrows) emerging at the end of the second circle of the first-line chemotherapy. (C) A significant response to the whole brain radiation is observed.

Abbreviation: MR, magnetic resonance. 
mass located elsewhere in the body. Findings indicate that symptomatic ocular metastasis could be an early event, and a duration of even 3 months, as seen in our patient, could cause rapid deterioration of the condition. Patients who have been diagnosed with orbital metastases usually carry a poor prognosis: the mean survival after diagnosis is 14-18 months, ${ }^{8,9,11}$ which remains the same as nearly that observed half a century earlier.

A variety of tumors and pseudotumors can involve the orbit. In a series of 1,264 orbital space-occupying lesions, malignant tumors accounted for $38 \%$ of the lesions. The authors concluded that with the knowledge of the incidence of the various tumors and pseudotumors, combined with improvement in imaging, complete history, and ophthalmologic examinations, clinicians should be able to reach a considerably accurate diagnosis. ${ }^{4}$ Usually, ophthalmologists play an important role in the diagnosis and differential diagnosis of metastatic orbital cancer by providing valuable information. The most frequent symptoms at presentation may include the diplopia (48\%), pain (42\%), proptosis (26\%), and decreased vision $(16 \%-30 \%)$, which are similar to other orbital tumors or inflammatory pseudotumor. ${ }^{8,10,11}$ In our patient, the ocular symptom of vision change was probably due to the tumor invasion of the optic nerve, leading to visual impairment. If a patient presents with orbital signs with a suspicion of malignant cancer, the diagnosis of orbital metastasis should prompt a systemic investigation to detect a primary lesion and other sites of metastasis. Provisional diagnosis of the orbital metastatic sites by imaging is important for guiding biopsy and staging. In series, CT had a better evaluation of bone and could identify metastases within the orbital fat, mostly located in the extraconal space. ${ }^{7,8} \mathrm{MRI}$ is the imaging method of choice in diagnoses of orbital lesions, showing a higher soft tissue contrast, and it also has the advantage of lack of exposure to ionizing radiation over $\mathrm{CT}$. The characteristic identity of metastatic lesions on MRI is an inhomogeneous low signal mass on $\mathrm{T} 1$ images, with increased signal intensity on T2 images. ${ }^{9}$ A previous report demonstrated the advantages of fluorodeoxyglucose positron emission tomography/ $\mathrm{CT}$ in detecting the primary site and in defining the total extent of disseminated disease. ${ }^{13}$ Sufficient imaging followed by a biopsy will usually confirm the diagnosis. Routine surgical biopsy needs special considerations. If the manifestation mimics orbital inflammation, a lymphoproliferative disorder, or other unknown orbital lesion, or occurs without a history of a systemic cancer, an open biopsy is preferred. However, when a systemic malignancy has been determined previously or when the lesion is easily accessible and the patient refuses an open biopsy, FNAB can be useful as a minimally invasive procedure. Compared to open biopsy, FNAB is minimally invasive and provides quick results. ${ }^{14} \mathrm{~A}$ useful supplement or an alternative to FNAB, which is cytological, is core needle biopsy, aimed at obtaining an adequate tissue sample for histological diagnosis, which reached a diagnostic accuracy of $94 \%$ in previous reports. ${ }^{15}$ The results of biopsy can provide more conclusive diagnosis, but also carry a high risk of severe complications, especially the damage to the eye globe, optic nerve, vessels, and even the needle-track tumor seeding simultaneously. ${ }^{16}$ Sometimes, the patient's basic condition is poor and radiological examinations can help identify the metastatic lesions, in which case an invasive biopsy can be avoided. The risk of a procedure should always be weighed against the benefits. ${ }^{4}$ In our case, given the burden of rapid process and the high risk of potential damage to vision, the patient required urgent antitumor management and refused another invasive procedure. Therapy targeting the primary lung cancer must take precedence.

Treatment for orbital pulmonary metastases is usually palliative, with the intent of both local tumor control and preservation of vision. Systemic chemotherapy has proved curative effect in most cases, but radiotherapy may be the mainstay for orbital metastases, with objective response rates up to $79 \%$ and the ability to save the quality of vision in $80 \%$ of cases without an invasive procedure, which can decompress the compressive optic nerve. ${ }^{17,18}$ The typical dose of radiotherapy is 30-50 Gy, which should be given with caution so as to prevent eyelashes loss and lacrimal apparatus injury. The cornea and scleral eye wall are resistant, but the lens is very sensitive to radiation and can be easily damaged and cause further deterioration in vision. Other management options such as observation and hormone administration depend on clinical circumstances. Hormone therapy is mainly used in breast or prostate cancer. Surgery is usually recommended only in selected patients, aiming at symptom relief and improvement of orbital function as long as possible; but in general, this is not the therapy of first choice. Recently, novel antitumor agents have had proven efficacy in patients with advanced NSCLC, and their integration into new treatment has evoked the exploration for orbital metastases; in fact, Zarogoulidis et $\mathrm{al}^{19}$ reported favorable efficacy of a combination chemotherapy of bevacizumab and pemetrexed in patients with orbital metastasis. Bevacizumab's efficacy in radiation retinopathy has also been reported. ${ }^{20}$ In contrast to traditional cytotoxic agents, the capability of TKIs to permeate across blood-brain barrier is certain and has been proven to boost cerebrospinal fluid exposure in refractory metastases. ${ }^{21,22}$ Are TKIs capable of rapidly permeating across brain-ocular barrier to increase the local drug concentration 
to overcome the resistance of refractory metastases? Yasuko et $\mathrm{al}^{23}$ reported a case of a patient with NSCLC and EGFRactivating mutation in whom a marked positive response to gefitinib therapy was shown in both the primary lesion and orbital metastasis. The eye functions as a vital organ to the quality of our life, and its special structure needs specifically designed agents, so that if the small-molecular inhibitors of TKI can improve the molecular response with limited drug toxicity in treatment of orbital metastases, it does deserve to be elucidated by further study.

\section{Conclusion}

The most challenging aspect of this case for diagnosis is the rarity and scant knowledge about it, but there is a real incidence of orbital metastasis being the initial presentation of lung adenocarcinoma. A large index of suspicion is essential when an orbital lesion appears, because an early, prompt intervention is vital to protect vision and maintain quality of life. Imaging studies can be used to characterize the orbital metastases for obtaining the provisional diagnosis. The choice of a biopsy procedure should be fully weighed for the cost/benefit ratio. Novel drugs, especially the TKIs, brighten the treatment for advanced lung cancer patients, but the efficacy in orbital metastases needs stronger evidence.

\section{Acknowledgments}

This study was supported by the National Nature Science Foundation of China grant (8147810), the special fund for Scientific Research in Public Interest (201402011) and the scientific research foundation of Shandong province of Outstanding Young Scientist Award (BS2013YY040).

\section{Disclosure}

The authors report no conflicts of interest in this work.

\section{References}

1. Siegel RL, Miller KD, Jemal A. Cancer statistics, 2015. CA Cancer J Clin. 2015;65(1):5-29.

2. Hess KR, Varadhachary GR, Taylor SH, et al. Metastatic patterns in adenocarcinoma. Cancer. 2006;106(7):1624-1633.

3. Ahmad SM, Esmaeli B. Metastatic tumors of the orbit and ocular adnexa. Curr Opin Ophthalmol. 2007;18(5):405-413.

OncoTargets and Therapy

\section{Publish your work in this journal}

OncoTargets and Therapy is an international, peer-reviewed, open access journal focusing on the pathological basis of all cancers, potential targets for therapy and treatment protocols employed to improve the management of cancer patients. The journal also focuses on the impact of management programs and new therapeutic agents and protocols on

Submit your manuscript here: http://www.dovepress.com/oncotargets-and-therapy-journal
4. Shields JA, Shields CL, Scartozzi R. Survey of 1,264 patients with orbital tumors and simulating lesions: the 2002 Montgomery Lecture, part 1. Ophthalmology. 2004;111(5):997-1008.

5. Bonavolonta G, Strianese D, Grassi P, et al. An analysis of 2,480 space-occupying lesions of the orbit from 1976 to 2011. Ophthal Plast Reconstr Surg. 2013;29(2):79-86.

6. Gonzalez F, Lopez-Couto C. Orbital metastases. A report of four cases and a review of the literature. Arch Soc Esp Oftalmol. 2006;81(8):451-462.

7. Holland D, Maune S, Kovacs G, Behrendt S. Metastatic tumors of the orbit: a retrospective study. Orbit. 2003;22(1):15-24.

8. Valenzuela AA, Archibald CW, Fleming B, et al. Orbital metastasis: clinical features, management and outcome. Orbit. 2009;28(2-3):153-159.

9. Yan J, Gao S. Metastatic orbital tumors in southern China during an 18-year period. Graefes Arch Clin Exp Ophthalmol. 2011;249(9):1387-1393.

10. Amemiya T, Hayashida H, Dake Y. Metastatic orbital tumors in Japan: a review of the literature. Ophthal Epidemiol. 2002;9(1):35-47.

11. Shields JA, Shields CL, Brotman HK, Carvalho C, Perez N, Eagle RC Jr. Cancer metastatic to the orbit: the 2000 Robert M. Curts Lecture. Ophthal Plast Reconstr Surg. 2001;17(5):346-354.

12. Mori H, Maekawa N, Satoda N, Otsuka N, Sakai N, Fukuse T. [A case of primary lung cancer with initial symptoms due to orbital metastases]. Nihon Kokyuki Gakkai zasshi. 2003;41(1):19-24. Japanese.

13. Manohar K, Mittal BR, Bhattacharya A, Gupta A. Orbital metastases as presenting sign of lung carcinoma: detection of primary malignancy and disease burden by F-18 FDG PET/CT. Nucl Med Mol Imaging. 2012;46(1):73-75.

14. Singhal N, Mundi IK, Handa U, Punia RP, Mohan H. FNA in diagnosis of orbital lesions causing proptosis in adults. Diagn Cytopathol. 2012; 40(10):861-864.

15. Gong Y, Sneige N, Guo M, Hicks ME, Moran CA. Transthoracic fine-needle aspiration vs concurrent core needle biopsy in diagnosis of intrathoracic lesions: a retrospective comparison of diagnostic accuracy. Am J Clin Pathol. 2006;125(3):438-444.

16. Yarovoy AA, Bulgakova ES, Shatskikh AV, Uzunyan DG, Kleyankina SS, Golubeva OV. CORE needle biopsy of orbital tumors. Graefes Arch Clin Exp Ophthalmol. 2013;251(8):2057-2061.

17. Ratanatharathorn V, Powers WE, Grimm J, et al. Eye metastasis from carcinoma of the breast: diagnosis, radiation treatment and results. Cancer Treat Rev. 1991;18(4):261-276.

18. Char DH, Miller T, Kroll S. Orbital metastases: diagnosis and course. Br J Ophthalmol. 1997;81(5):386-390.

19. Zarogoulidis P, Terzi E, Kouliatsis G, et al. Orbital metastases as the first manifestation of lung adenocarcinoma. Case Rep Ophthalmol. 2011;2(1):34-38.

20. Finger PT, Chin K. Anti-vascular endothelial growth factor bevacizumab (avastin) for radiation retinopathy. Arch Ophthalmol. 2007; 125(6):751-756.

21. Zhang J, Yu J, Sun X, Meng X. Epidermal growth factor receptor tyrosine kinase inhibitors in the treatment of central nerve system metastases from non-small cell lung cancer. Cancer Lett. 2014;351(1):6-12.

22. Mitsudomi T, Morita S, Yatabe Y, et al. Gefitinib versus cisplatin plus docetaxel in patients with non-small-cell lung cancer harbouring mutations of the epidermal growth factor receptor (WJTOG3405): an open label, randomised phase 3 trial. Lancet Oncol. 2010;11(2):121-128.

23. Koma Y, Goto K, Yoshida C, et al. Orbital metastasis secondary to pulmonary adenocarcinoma treated with gefitinib: a case report. J Med Case Rep. 2012;6:353.

\section{Dovepress}

patient perspectives such as quality of life, adherence and satisfaction. The manuscript management system is completely online and includes a very quick and fair peer-review system, which is all easy to use. Visit $\mathrm{http}: / /$ www.dovepress.com/testimonials.php to read real quotes from published authors. 